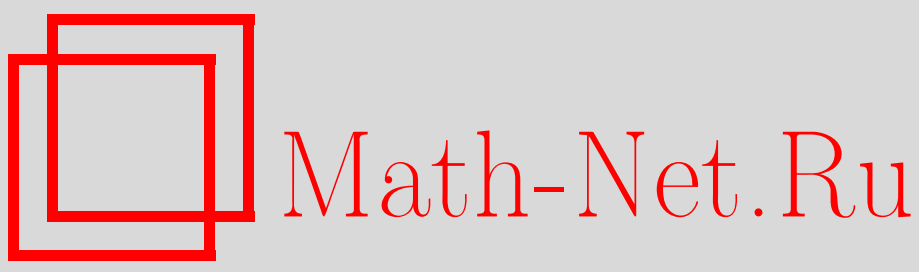

Н. Б. Енгибарян, Уравнения в свертках, содержащие сингулярные вероятностные распределения, Изв. РАН. Сер. матем., 1996, том 60, выпуск 2, 21-48

DOI: https://doi.org/10.4213/im70

Использование Общероссийского математического портала Math-Net.Ru подразумевает, что вы прочитали и согласны с пользовательским соглашением

http://www . mathnet.ru/rus/agreement

Параметры загрузки:

IP: 3.80 .181 .102

26 апреля 2023 г., 03:23:54 
УДК 517.9

\author{
Н. Б. Енгибарян
}

\title{
Уравнения в свертках, содержащие сингулярные вероятностные распределения
}

Работа посвящена уравнениям вида

$$
\varphi(x)=g(x)-\int_{0}^{\infty} \varphi(t) d T(x-t),
$$

где $T$ - непрерывная функция ограниченной вариации на $(-\infty ; \infty)$, содержащая сингулярную компоненту. Сначала изучаются асимптотические и другие свойства решения формально вольтерровых уравнений $(*)$, соответствующих $T(x)=0$ при $x \leqslant 0$. Далее выводятся и изучаются нелинейные уравнения факторизации (НУ $\Phi)$ для $(*)$. Факторизация строится в случае, когда $T(-\infty)=0$, $T(x) \uparrow$ по $x, T(+\infty)=\mu \leqslant 1$. С помощью этой факторизации доказываются теоремы существования для однородного $(g=0)$ и неоднородного уравнений в особом случае $\mu=1$.

Библиография: 14 наименований.

\section{Введение}

Рассмотрим интегральное уравнение

$$
\varphi(x)=g(x)-\int_{0}^{\infty} \varphi(t) d T(x-t),
$$

где $T$ - заданная функция ограниченной вариации на $\mathbb{R}$ :

$$
\mu \equiv \bigvee_{-\infty}^{+\infty} T<+\infty
$$

Функцию $T$ назовем предбядром уравнения (0.1). Интеграл в правой части (0.1) понимается в смысле Римана-Стилтьеса.

Согласно разложению Лебега имеем [1]

$$
T=T_{A}+T_{D}+T_{S}
$$

$T_{A}$ - абсолютно непрерывная компонента функции $T$. Она почти всюду имеет конечную производную $T^{\prime}(x)=K(x) \in L_{1}(\mathbb{R})$ и восстанавливается с ее помощью:

$$
T(x)=\int_{-\infty}^{x} K(t) d t+C .
$$

Дискретная компонента $T_{D}$ в $(0.3)$ является функцией скачков с конечной суммой абсолютных величин скачков. Сингулярная компонента $T_{S}$ является сингулярной 
функцией (или нулем), т.е. $T_{S}$ - непрерывная функция ограниченной вариации, имеющая почти всюду равную нулю производную. Классическим примером сингулярной функции является канторова лестница [1].

В дальнейшем индексами $A, D$ и $S$ будут отмечены соответствующие компоненты данной функции ограниченной (или локально ограниченной) вариации, а индексом $C$ - непрерывная компонента: $T_{C}=T_{A}+T_{S}$. Отметим, что в случае неубывающей функции $T$ ее компоненты $T_{A}, T_{D}$ и $T_{S}$ также неубывающие.

Важным частным случаем уравнения (0.1) является интегральное уравнение Винера-Хопфа второго рода (ИУВХ): если $T=T_{A}$, то

$$
\varphi(x)=g(x)+\int_{0}^{\infty} K(x-t) \varphi(t) d t .
$$

Если $T(x)=$ const при $x \leqslant 0$, то $(0.1)$ обрашается в уравнение восстановления $(\mathrm{YB})$ :

$$
\varphi(x)=g(x)-\int_{0}^{x} \varphi(t) d T(x-t) .
$$

ИУВХ (0.4) и соответствуюшее УВ имеют широкое применение в теории переноса излучения, в кинетической теории газов, в теории вероятностей и др. Они очень хорошо изучены (см. [2]-[10]). Достаточно изучены их интегрально-разностные аналоги, соответствуюшие случаю $T=T_{A}+T_{D}$ (см. [7], [6], [9], [11]).

Одной из важных задач теории вероятностей является следующая (см. [4]): для заданной случайной величины $\eta$ найти независимо распределенную случайную величину $\zeta$ такую, чтобы имело место соотношение

$$
\zeta \sim(\eta+\zeta)_{+}
$$

Эквивалентность двух случайных величин понимается в смысле совпадения их функций распределения. Через $\eta_{+}$обозначается следующая случайная величина: $\eta_{+}=\eta$ при $\eta \geqslant 0$ и $\eta_{+}=0$ при $\eta<0$.

Соотношение (0.6) сводится к однородному интегральному уравнению

$$
S(x)=-\int_{0}^{\infty} S(t) d T(x-t),
$$

где $T$ - функция распределения $\eta$, а $S$ - искомая функция распределения $\zeta$. Как в $(0.7)$, так и в других приложениях уравнения (0.1) по теории вероятностей роль функции $T$ обычно играет функция распределения некоторой случайной величины. Тогда

$$
T(-\infty)=0, \quad T(+\infty)=1, \quad T(x) \uparrow \quad \text { по } x .
$$

В случае наличия сингулярной компоненты функции $T$ уравнение $(0.1)$ почти не изучено. Аналогично обстоит дело с сингулярными распределениями в теории вероятностей. В [12, с. 177] сказано: “Сингулярные распределения не поддаются аналитическому изучению... (они) однако играют важную принщипиальную роль, и многие статистические тесты основываются на их существовании".

Настояшая работа посвящена уравнению (0.1) и его некоторым частным случаям, когда предъядро $T$ непрерывно и обладает сингулярной компонентой: $T=$ 
$T_{C}=T_{A}+T_{S}$. Основные результаты работы по уравнению $(0.1)$ относятся к случаю, когда $T=\mu T_{0}$, где $0<\mu \leqslant 1$, а $T_{0}$ удовлетворяет условиям вида (0.8). Что касается уравнения (0.5), то оно будет изучено при более слабом ограничении на $T$, а именно $\bigvee_{0}^{r} T<+\infty$ при некотором $r \leqslant+\infty$.

Предлагаемый подход является развитием метода нелинейных уравнений факторизачии (НУФ) автора (см. [5], [10], [13]). Выводится и изучается НУ $\Phi$ для уравнения (0.1). Это уравнение факторизации одновременно является новой формой НУФ интегральных операторов Винера-Хопфа (см. [7], [10]). Сочетание метода НУ $\Phi$ и других специальных методов позволяет построить достаточно полную теорию однородного и неоднородного уравнений $(0.1),(0.8)$.

\section{§1. Вспомогательные предложения}

Уравнения (0.1) и (0.5) мы будем рассматривать в специальных классах непрерывных или кусочно непрерывных функций.

1. Функциональные пространства. Введем в рассмотрение следующие функциональные банаховы пространства и классы функций:

a) банахово пространство $C[a ; b]$ непрерывных на $[a ; b] \subset \mathbb{R}$ функций с равномерной нормой $\|f\|=\max _{x \in[a ; b]}|f(x)|$; через $\omega(\delta, f)$, как обычно, обозначается модуль непрерьвности функции $f \in C[a ; b]$ :

$$
\omega(\delta, f)=\sup _{\left|x_{1}-x_{2}\right|<\delta}\left|f\left(x_{1}\right)-f\left(x_{2}\right)\right| ;
$$

б) банахово пространство $C_{0}[0 ; \infty) \equiv C[0 ; \infty]$ непрерывных на $[0 ; \infty)$ функций, имеюших конечный предел в $\infty$, с нормой $\|f\|=\sup |f(x)|$;

в) банахово пространство $V[a ; b]$ функций ограниченной вариации на $[a ; b] \subset \mathbb{R}$ с нормой

$$
\|f\|=|f(a)|+\bigvee_{a}^{b} f
$$

г) банахово пространство $V_{C}[a ; b] \subset V[a ; b]$ непрерывных функций ограниченной вариации на $[a ; b]$ с нормой (1.1) (из сходимости по норме (1.1) следует равномерная сходимость);

д) классы $\operatorname{Mon}[a ; b]$ и $\operatorname{Mon}^{C}[a ; b]$ неубывающих ограниченных функций (они образуют воспроизводящие конусы в своих пространствах: $V[a ; b]$ и $V_{C}[a ; b]$ соответственно);

е) банахово пространство $H_{\alpha}[a ; b] \subset C[a ; b]$ функций, удовлетворяюших условию Гёльдера-Липшица с показателем $\alpha, 0<\alpha \leqslant 1$; норма в нем определяется следуюшим образом:

$$
\|f\|=|f(a)|+\sup _{x_{1} \neq x_{2}} \frac{\left|f\left(x_{1}\right)-f\left(x_{2}\right)\right|}{\left|x_{1}-x_{2}\right|^{\alpha}}
$$

ж) пусть $E[a ; r], r \leqslant+\infty,-$ одно из пространств $C[a ; r], V_{C}[a ; r], V[a ; r]$ и $E^{+}=E[0 ; \infty]$; обозначим через $E^{\mathrm{loc}}[0 ; \infty)$ топологическое пространство определенных на $[0 ; \infty)$ функций, принадлежащих $E[0 ; r] \forall r<+\infty$. Сходимость в $E^{\text {loc }}$ определяется как сходимость по $E[0 ; r] \forall r<+\infty$. 
2. Об интеграле Стилтьеса. Рассмотрим интеграл

$$
\alpha=\int_{a}^{b} f(x) d \sigma(x), \quad-\infty \leqslant a<b \leqslant+\infty,
$$

где $\sigma \in V_{C}[a ; b]$, a $f \in C[a ; b]$, причем сушествуют конечные пределы $f(a+)$ и $f(b-)$. Тогда рассматриваемый интеграл существует как собственный или несобственньй интеграл Стилтьеса [1].

Пусть $h(x)=\bigvee_{a}^{x} \sigma$. Из непрерывности $\sigma$ следует непрерывность $h$ на $[a ; b]$. Имеет место неравенство

$$
|\alpha| \leqslant \int_{a}^{b}|f(x)| d h(x) \equiv \int_{a}^{b}|f(x)||d \sigma(x)|
$$

Мы воспользуемся формулой интегрирования по частям:

$$
\int_{a}^{b} f(x) d \sigma(x)=f(b) \sigma(b)-f(a) \sigma(a)-\int_{a}^{b} \sigma(x) d f(x) .
$$

В случае отсутствия дискретной компоненты $\sigma_{D}$ у функции $\sigma$ интеграл Стилтьеса обладает свойством аддитивности.

Мы воспользуемся также теоремой Фубини о замене порядка интегрирования в кратном интеграле и первой теоремой Хелли о предельном переходе под знаком интеграла Стилтьеса (см. [1]).

\section{§2. Простейшие свойства операторов свертки с непрерывным предъядром}

1. Классы операторов. Введем в рассмотрение классы $\Omega_{C}, \Omega_{C}^{ \pm} \subset \Omega_{C}$ и $\Omega_{C, r}^{+}$ операторов типа свертки:

$$
\begin{aligned}
& \mathbb{U}^{+} \in \Omega_{C, r}^{+}, \quad \text { если } \quad \mathbb{U}^{+} \varphi(x)=-\int_{0}^{x} \varphi(t) d U(x-t), \quad U \in V_{C}[0 ; r], \\
& r \leqslant+\infty ; \quad \Omega_{C}^{+} \equiv \Omega_{C, \infty}^{+} ; \\
& \mathbb{U}^{-} \in \Omega_{C}^{-}, \quad \text { если } \quad \mathbb{U}^{-} \varphi(x)=\int_{x}^{\infty} \varphi(t) d U(t-x), \quad U \in V_{C}^{+} ; \\
& \mathbb{T} \in \Omega_{C}, \quad \text { если } \quad \mathbb{T} \varphi(x)=-\int_{0}^{\infty} \varphi(t) d T(x-t), \quad T \in V_{C}(\mathbb{R}) .
\end{aligned}
$$

Через $\Omega_{A}^{ \pm}$и $\Omega_{A}$ обозначаются аналогичные классы операторов, соответствующие абсолютно непрерывным предъядрам. Формально вольтерровы операторы из классов $\Omega_{C, r}^{+}$и $\Omega_{C}^{-}$назовем соответственно нижними и верхними операторами восстановления.

Предъядра рассматриваемых операторов $\mathbb{U}^{ \pm}$и $\mathbb{T}$ определяются с точностью до постоянных слагаемых. $T$ мы будем нормировать согласно условию $T(-\infty)=0$, а $U$ - одним из следуюших двух способов: либо $U(0)=0$, либо $U(\infty)=0$. 
2. Класс $\Omega_{C, r}^{+}$. Пусть $\mathbb{U}^{+} \in \Omega_{C, r}^{+}$и $U(0)=0$. Обозначим

$$
\gamma_{r}=\bigvee_{0}^{r} U<+\infty, \quad \gamma=\gamma_{\infty}
$$

Лемма 2.1. а) Oператор $\mathbb{U}^{+}$действует в пространствах $E[0 ; r]$, причем во всех этих пространствах

$$
\left\|\mathbb{U}^{+}\right\| \leqslant \gamma_{r} .
$$

б) Если $U \in \operatorname{Mon}^{C}[0 ; r]$, mо $\mathbb{U}^{+}$переводит Mon $[0 ; r] u \operatorname{Mon}^{C}[0 ; r]$ в себя, m.e. является положительным оператором в пространствах $V[0 ; r]$ u $V_{C}[0 ; r]$.

ДокАЗАТЕльСтво. Пусть $\psi=\mathbb{U}^{+} \varphi$. Имеем

$$
\psi(x)=-\int_{0}^{x} \varphi(t) d U(x-t) .
$$

Существование функции $\psi$ при $x \in[0 ; r]$ следует из общих свойств интеграла Стилтьеса. Путем замены переменной $x-t=y$ получаем

$$
\psi(x)=\int_{0}^{x} \varphi(x-y) d U(y)
$$

Из (2.3) непосредственно следует, что если $U \in \operatorname{Mon}^{C}[0 ; r]$ и $\varphi \in \operatorname{Mon}[0 ; r]$ или $\varphi \geqslant 0$, то и $\psi \in \operatorname{Mon}[0 ; r]$ или $\psi \geqslant 0$. Пусть теперь $U \in V_{C}[0 ; r]$ и $\varphi \in$ $V[0 ; r]$. Представив каждую из этих функций в виде разности двух монотонных функций, мы получаем представление $\psi$ в виде комбинации четырех монотонных ограниченных функций, поэтому $\psi \in V[0 ; r] ;$ стало быть, $\mathbb{U}$ переводит $V[0 ; r]$ в себя. Предположим теперь, что $\varphi \in C[0 ; r], \quad r<+\infty$. Для $\delta>0$ и $x, x+\delta \in[0 ; r]$ имеем

$\psi(x+\delta)-\psi(x)=\int_{0}^{x}[\varphi(x+\delta-y)-\varphi(x-y)] d U(y)+\int_{x}^{x+\delta} \varphi(x+\delta-y) d U(y)$.

Используя оценку (1.2), получаем

$$
|\psi(x+\delta)-\psi(x)| \leqslant \omega(\delta, \varphi) \bigvee_{0}^{x} U+\|\varphi\|_{C[0 ; r]} \int_{x}^{x+\delta}|d U(y)|,
$$

откуда

$$
\omega(\delta, \psi) \leqslant \gamma_{r} \omega(\delta, \varphi)+\omega(\delta, h)\|\varphi\|_{C[0 ; r]},
$$

где $h(x)=\bigvee_{0}^{x} U \in C[0 ; r]$. Из оценки (2.4) следует $\psi \in C[0 ; r]$. Оценка $(2.1)$ в случае пространства $C[0 ; r]$ очевидна.

Пусть теперь $\varphi \in V_{C}[0 ; r]$. Согласно вьшедоказанному $\psi \in V[0 ; r]$ и $\psi \in C[0 ; r]$, поэтому $\psi \in V_{C}[0 ; r]$.

Докажем оценку $(2.1)$ в $V[0 ; r]$ и $V_{C}[0 ; r], r<+\infty$. Пусть $\varphi \in V[0 ; r]$ и П некоторое разбиение промежутка $[0 ; r]: \Pi: 0=x_{0}<x_{1}<\cdots<x_{n}=r$, а $\lambda-$ диаметр этого разбиения. 
Обозначим $\psi_{k}=\psi\left(x_{k}\right), h_{k}=h\left(x_{k}\right)$, где $h(x)=\bigvee_{0}^{x} U$. Полагая в $(2.4) x=x_{k}$ и $x+\delta=x_{k+1}(k=\overline{0, n-1})$, получаем

$$
\psi_{k+1}-\psi_{k}=\int_{0}^{x_{k}}\left[\varphi\left(x_{k+1}-y\right)-\varphi\left(x_{k}-y\right)\right] d U(y)+\int_{x_{k}}^{x_{k+1}} \varphi\left(x_{k+1}-y\right) d U(y),
$$

откуда с учетом неравенства $\left|\varphi\left(x_{k+1}-y\right)\right| \leqslant|\varphi(0)|+\omega(\lambda, \varphi)$ при $y \in\left[x_{k} ; x_{k+1}\right]$ получаем

$$
\begin{aligned}
\left|\psi_{k+1}-\psi_{k}\right| \leqslant & {[\omega(\lambda, \varphi)+|\varphi(0)|]\left(h_{k+1}-h_{k}\right) } \\
& +\sum_{m=0}^{k} \int_{x_{m}}^{x_{m+1}}\left|\varphi\left(x_{k+1}-y\right)-\varphi\left(x_{k}-y\right)\right| d h(y) .
\end{aligned}
$$

Суммируя эти неравенства по $k$ от 0 до $n-1$ и меняя порядок суммирования в двойной сумме по $k$ и $m$, получаем

$$
\begin{aligned}
& \sum_{k=0}^{n-1}\left|\psi_{k+1}-\psi_{k}\right| \leqslant[|\varphi(0)|+\omega(\lambda, \varphi)] h(r) \\
& \quad+\sum_{m=0}^{n-1} \sum_{k=m}^{n-1}\left|\varphi\left(x_{k+1}-y\right)-\varphi\left(x_{k}-y\right)\right| d h(y) \\
& \quad \leqslant[|\varphi(0)|+\omega(\lambda, \varphi)] h(r)+\sum_{m=0}^{n-1} \int_{x_{m}}^{x_{m+1}}(\underset{0}{\bigvee} \varphi) d h(y)
\end{aligned}
$$

откуда

$$
\sum_{k=0}^{n-1}\left|\psi_{k+1}-\psi_{k}\right| \leqslant[|\varphi(0)|+\omega(\lambda, \varphi)] \bigvee_{0}^{r} U+\left(\bigvee_{0}^{r} \varphi\right)\left(\bigvee_{0}^{r} U\right)
$$

В силу произвольности разбиения П (и произвольной малости $\lambda$ ) из (2.6) приходим к неравенству

$$
\bigvee_{0}^{r} \psi \leqslant\left(|\varphi(0)|+\bigvee_{0}^{r} \varphi\right) \bigvee_{0}^{r} U
$$

Это неравенство с учетом $\psi(0)=0$ представляет собой оценку $(2.1)$ в случае пространств $V[0 ; r]$ и $V_{C}[0 ; r]$.

Итак, все утверждения леммы при $r<+\infty$ доказаны. Рассмотрим теперь случай $r=+\infty$. Покажем сначала, что при $U \in V_{C}^{+}$оператор $\mathbb{U}^{+}$переводит $C[0 ; \infty]$ в себя. Пусть $\varphi \in C[0 ; \infty]$ и $\varphi(\infty)=\alpha$. Покажем, что

$$
\lim _{x \rightarrow+\infty} \psi(x)=\beta \equiv \alpha \int_{0}^{\infty} d U(x)=\alpha U(+\infty),
$$

т.е. в (2.3) можно совершить формальньй предельньй переход при $x \rightarrow+\infty$. Пусть $M=\sup |\varphi(x)|$ и $\varepsilon>0$. Выберем число $r>0$ такое, чтобы выполнялись неравенства

$$
|\varphi(x)-\alpha|<\frac{\varepsilon}{2 \gamma_{+}}, \quad x>r, \quad \text { и } \quad(\alpha+M) \int_{r}^{\infty}|d U(x)|<\frac{\varepsilon}{2} .
$$


Имеем

$$
\begin{aligned}
|\psi(x)-\beta|= & \mid \int_{0}^{r}[\varphi(x-t)-\alpha] d U(t) \\
& +\int_{r}^{x} \varphi(x-t) d U(t)-\int_{r}^{\infty} \alpha d U(t) \mid<\varepsilon, \quad x>2 r .
\end{aligned}
$$

Оценка (2.1) при $r=+\infty$ непосредственно следует из справедливости этой оценки $\forall r<+\infty$. Лемма доказана.

ЗАмечание. Оператор $\mathbb{U}^{+} \in \Omega_{C, r}^{+}$отображает пространства $E[0 ; r]$ в свои подпространства, состояшие из функций, для которых $\psi(0)=0$. Поэтому $\mathbb{U}^{+}$ действует в этих подпространствах $E_{0}[0 ; r]$.

При $\varphi(x) \equiv 1$ из $(2.3)$ имеем $\psi(x)=U(x)$. Этот пример показывает, что гладкость функции $\psi$ может быть не выше гладкости $U$, сколь гладкой бы ни была функция $\varphi$. Если же оператор $\mathbb{U}^{+}$рассмотреть в подпространствах $E_{0}[0 ; r]$, то свойства гладкости $\varphi$ переходят к $\psi$.

ЛЕмма 2.2. Если $\mathbb{U}^{+} \in \Omega_{C, r}^{+}$, а функиия $\varphi$ непрерывно дифференцируема или принадлежит $H_{\alpha}[0 ; r]$, причем $\varphi(0)=0$, то таковой является функиия $\psi=\mathbb{U}^{+} \varphi$, определенная согласно (2.2).

ДокАЗАТЕЛЬСТво. Пусть $\varphi \in C_{1}[0 ; r]$. Из $(2.3)$ с учетом $\varphi(0)=0$ имеем

$$
\begin{aligned}
\psi(x+\Delta x)-\psi(x)= & \int_{0}^{x}[\varphi(x+\Delta x-t)-\varphi(x-t)] d U(t) \\
& +\int_{x}^{x+\Delta x}[\varphi(x+\Delta x-t)-\varphi(0)] d U(t) .
\end{aligned}
$$

Во втором интеграле аргумент $x+\Delta x-t$ изменяется в пределах от 0 до $\Delta x$. Поэтому

$$
\varphi(x+\Delta x-y)-\varphi(0)=\Delta x \cdot \varphi^{\prime}\left(\theta_{1} \Delta x\right) \theta_{2}, \quad 0 \leqslant \theta_{1,2} \leqslant 1 .
$$

Из полученных вьше соотношений легко следует, что

$$
\psi^{\prime}(x)=\int_{0}^{x} \varphi^{\prime}(x-y) d U(y) \in C[0 ; r]
$$

поэтому $\psi \in C_{1}[0 ; r]$. Аналогично доказывается, что $\psi \in H_{\alpha}[0 ; r]$ при $\varphi \in H_{\alpha}[0 ; r]$ и $\varphi(0)=0$.

3. Единственность предъядра. Покажем, что операторы $\mathbb{U}_{1}^{+}$и $\mathbb{U}_{2}^{+}$из $\Omega_{C, r}^{+}$ совпадают в рассматриваемых пространствах тогда и только тогда, когда их предъядра отличаются на постоянное слагаемое.

Пусть $\mathbb{U}^{+}=\mathbb{U}_{1}^{+}-\mathbb{U}_{2}^{+}$. Его предъядром служит функция $U=U_{1}-U_{2}$. Пусть $\int_{0}^{x} \varphi(t) d U(x-t)=0 \quad \forall \varphi \in V_{C}[0 ; r]$. При $\varphi(x) \equiv 1$ имеем $\int_{0}^{x} d U(x)=0$, т.е. $U(x)=$ const. 
4. Свертка в $V_{C}[0 ; r]$. С помощью интеграла (2.3) определяется операция свертки в пространстве $V_{C}[0 ; r], r \leqslant+\infty$, и в пространстве $V_{C}^{0}[0 ; r]$, состоящем из функций, удовлетворяющих условию $\varphi(0)=0$.

Пусть $f, \varphi \in V_{C}^{0}[0 ; r]$. Их сверткой назовем функцию

$$
(f * \varphi)(x)=\int_{0}^{x} f(x-t) d \varphi(t) .
$$

В $V_{C}[0 ; r]$ свертка определяется следуюшим образом:

$$
(f * \varphi)(x)=\int_{0}^{x}[f(x-t)-f(0)] d \varphi(t) .
$$

Пространства $V_{C}[0 ; r]$ и $V_{C}^{0}[0 ; r]$, снабженные операцией умножения $*$, являются коммутативньми сверточными алгебрами без единицы. Из (2.6) следует, что

$$
\|f * \varphi\|_{V_{C}} \leqslant\|f\|_{V_{C}} \cdot\|\varphi\|_{V_{C}}
$$

т.е. $V_{C}[0 ; r]$ является банаховой алгеброй. Заметим, что при $f, \varphi \in \operatorname{Mon}^{C}[0 ; \infty)$ неравенство (2.10) обращается в равенство.

Отмеченные выше свойства свертки в пространстве $V_{C}$ аналогичны свойствам свертки $\int_{0}^{x} f(x-t) \varphi(t) d t$ в пространстве $L_{1}^{+}$и широко применяются в теории вероятностей.

5. Класс $\Omega_{C}^{-}$. Рассмотрим оператор $\mathbb{U}^{-} \in \Omega_{C}^{-}$, определенньй в п. 1 настоящего параграфа. Пусть $\psi=\mathbb{U}^{-} \varphi$. Имеем

$$
\psi(x)=\int_{0}^{\infty} \varphi(x+t) d U(t), \quad U \in V_{C}^{+} .
$$

Нетрудно проверить справедливость следующей леммы.

Лемма 2.3. Оператор $\mathbb{U}^{-}$действует в каждом из банаховых пространств $C[r ; \infty], V[r ; \infty], V_{C}[r ; \infty], H_{\alpha}[r ; \infty], \quad r \in \mathbb{R}$, причем в любом из указанных пространств

$$
\left\|\mathbb{U}^{-}\right\| \leqslant \gamma \equiv \bigvee_{0}^{\infty} U
$$

Ecлu $U \in \operatorname{Mon}^{C} u \varphi \in \operatorname{Mon}^{C}$, mo $\psi \in \operatorname{Mon}^{C} . \operatorname{Ec} \bumpeq u \varphi(\infty)=0$, mo $\psi(\infty)=0$.

Операторы из $\Omega_{C}^{-}$мы будем в основном рассматривать в пространствах функций, определенных на $[0 ; \infty)$.

Лемма 2.4. а) Пусть в (2.11) $\varphi \in V_{C}^{+} u h \in C[0 ; r]$. Тогда имеет место формула $($ при $r<+\infty)$

$$
\int_{0}^{r} h(x) d \psi(x)=\int_{0}^{\infty}\left[\int_{0}^{r} h(x) d \varphi(x+t)\right] d U(t) .
$$

б) Если $h \in \operatorname{Mon}[0 ; \infty)$ и сходится интеграл $\int_{0}^{\infty} h(x)|d \varphi(x)|$, то формула (2.13) справедлива также при $r=+\infty$. 
ДокаЗАТЕЛЬСтво. Пусть $h \in C[0 ; r]$. Интегрируя обе части равенства (2.11) по заряду $d h$ от 0 до $r<+\infty$ и используя теорему Фубини [1], получаем

$$
\int_{0}^{r} \psi(x) d h(x)=\int_{0}^{\infty}\left[\int_{0}^{r} \varphi(x+t) d h(x)\right] d U(t) .
$$

Применяя интегрирование по частям, будем иметь

$$
\begin{aligned}
\psi(r) h(r) & -\psi(0) h(0)-\int_{0}^{r} h(x) d \psi(x) \\
= & \int_{0}^{\infty}\left[\varphi(r+t) h(r)-\varphi(t) h(0)-\int_{0}^{r} h(x) d \varphi(x+t)\right] d U(t) .
\end{aligned}
$$

Отсюда с учетом равенств $\psi(0)=\int_{0}^{\infty} \varphi(t) d U(t)$ и $\psi(r)=\int_{0}^{\infty} \varphi(r+t) d U(t)$ приходим к формуле (2.13) при $r<+\infty$.

Утверждение б) леммы достаточно доказать для случая, когда $\varphi(t) \downarrow$ на $[0 ; \infty)$. Обозначим

$$
g_{r}(t)=\int_{0}^{r} h(x) d \varphi(x+t), \quad g(t)=g_{\infty}(t) .
$$

Имеем $g(t)=\int_{t}^{\infty} h(x-t) d \varphi(x)$. Из условий леммы следует, что функция $g(t) \downarrow 0$ при $t \rightarrow+\infty$. Имеем также $\lim _{r \rightarrow+\infty} g_{r}(t)=g(t)$ поточечно. Совершая в $(2.13)$ предельный переход при $r \rightarrow+\infty$, с учетом первой теоремы Хелли [1] приходим к утверждению б) леммы. Лемма доказана.

В конще пункта покажем, что операторы из $\Omega_{C}^{-}$совпадают тогда и только тогда, когда их предъядра отличаются на постоянное слагаемое. Действительно, пусть $\mathbb{U}_{1}^{-}, \mathbb{U}_{2}^{-} \in \Omega_{C}^{-}$и $\mathbb{U}^{-}=\mathbb{U}_{1}^{-}-\mathbb{U}_{2}^{-}$. Имеем

$$
\int_{0}^{\infty} \varphi(x+t) d U(t)=0
$$

Полагая $\varphi(x)=\varphi_{0}(x) \equiv \theta(y-x)$, где $\theta-$ функция Хевисайда, получим $U(y-x)=$ $U(\infty), x<y$, т.е. $\mathbb{U}^{-}=0$.

Наше утверждение доказано в пространстве $V^{+}$. Аппроксимируя по норме пространства $V^{+}$функцию $\varphi_{0}$ функциями $\varphi_{n}$ из $V_{C}^{+}$и заменяя $\varphi$ на $\varphi_{n}$, легко можно установить справедливость нашего утверждения в случае пространств $V_{C}^{+}$и $C[0 ; \infty]$.

6. Класс $\Omega_{C}$. Рассмотрим теперь оператор $\mathbb{T} \in \Omega_{C}$, определенный в п. 1 . Пусть $\psi=\mathbb{T} \varphi$. Имеем

$$
\psi(x)=-\int_{0}^{\infty} \varphi(t) d T(x-t)
$$

Используя аддитивность интеграла Стилтьеса при непрерывном $T$, из (2.14) будем иметь

$$
\mathbb{T}=\mathbb{T}_{+}+\mathbb{T}_{-},
$$

где

$$
\begin{aligned}
\left(\mathbb{T}_{+} \varphi\right)(x) & =-\int_{0}^{x} \varphi(t) d T_{+}(x-t), \\
\left(\mathbb{T}_{-} \varphi\right)(x) & =\int_{x}^{\infty} \varphi(t) d T_{-}(t-x), \\
T_{ \pm}(x) & = \pm[T( \pm x)-T(0)], \quad x \geqslant 0 .
\end{aligned}
$$


Покажем единственность представления (2.15). Достаточно показать, что пересечение подпространств $\Omega_{C}^{+} \cap \Omega_{C}^{-}$состоит только из нулевого оператора 0 . Пусть $\mathbb{U}^{ \pm} \in \Omega_{C}^{ \pm}$и $\mathbb{U}^{+}=\mathbb{U}^{-}$. Имеем

$$
\int_{0}^{\infty} \varphi(x+t) d U_{-}(t)=-\int_{0}^{x} \varphi(x-t) d U_{+}(t) .
$$

При $\varphi(x) \equiv 1$ получаем $U_{+}(x)-U_{+}(0)=U_{-}(0)-U_{-}(\infty)$, т.е. $U_{+}(x)=$ const. Поэтому $\mathbb{U}^{+}=\mathbb{U}^{-}=0$.

Нами показано, что $\Omega_{C}$ является прямой суммой своих подпространств $\Omega_{C}^{ \pm}$:

$$
\Omega_{C}=\Omega_{C}^{+} \oplus \Omega_{C}^{-} .
$$

Заметим, что при составлении суммы $\mathbb{U}=\mathbb{U}^{+}+\mathbb{U}^{-}$, где $\mathbb{U}^{ \pm} \in \Omega_{C}^{ \pm}$, мы должны позаботиться о непрерьвности предъядра $U$ в точке стыка 0 и о выполнении условия $U(-\infty)=0$. С учетом этих требований функция $U$ определяется следующим образом (при $\left.U_{ \pm}(0)=0\right)$ :

$$
U(x)=U_{-}(\infty)-U_{-}(-x), \quad x \leqslant 0, \quad U(x)=U_{+}(x)+U_{-}(\infty), \quad x>0 .
$$

Из представления (2.15) и лемм 2.1, 2.3 следует

Лемма 2.5. Onератор $\mathbb{T} \in \Omega_{C}$ действует в банаховы (m.е. $\left.C[0 ; \infty], V_{C}^{+}, V^{+}\right)$, причем в любом из әтих пространств

$$
\|\mathbb{T}\| \leqslant \mu \equiv \bigvee_{-\infty}^{\infty} T
$$

Eсли $T \in \operatorname{Mon}^{C}(\mathbb{R})$, mо $\mathbb{T}$ отображсает конус неотрицательньх функиий в себя.

Операторы из $\Omega_{C}$ мы будем рассматривать также в специальных классах функций из $C[0 ; \infty)$ и $V^{\text {loc }}[0 ; \infty)$, неограниченно растущих в $\infty$.

\section{§3. Уравнения восстановления}

1. Алгебры $\Omega_{C}^{ \pm}$. Хорошо известно, что класс интегральных операторов Винера-Хопфа $\Omega_{A}$ не замкнут относительно операторного умножения: ядро произведения двух интегральных операторов Винера-Хопфа может не быть зависящим только от разности аргументов. Классы же $\Omega_{A}^{ \pm}$образуют коммутативные алгебры. Аналогичная ситуация имеет место в случае классов $\Omega_{C}$ и $\Omega_{C}^{ \pm}$.

Ниже мы выведем правило композиции предъядер при умножении операторов из $\Omega_{C}^{+}$. Пусть $r \leqslant+\infty, \mathbb{U}_{k}^{+} \in \Omega_{C, r}^{+}(k=1,2)$,

$$
\mathbb{U}_{k}^{+} f(x)=-\int_{0}^{x} f(t) d U_{k}(x-t)
$$

$U_{k} \in V_{C}[0 ; r]$, a $f \in V_{C}[0 ; r]$. Представим $f$ в виде $f_{0}+f_{1}$, где $f_{0}(x) \equiv f(0)$, а $f_{1}(0)=0$. Обозначим $\varphi=\mathbb{U}_{1}^{+} f_{1}$ и $\psi=\mathbb{U}_{2}^{+} \varphi$. Имеем

$$
\varphi=U_{1} * f_{1}, \quad \psi=U_{2} * \varphi=U * f_{1}=\mathbb{U}^{+} f_{1},
$$

где $U=U_{2} * U_{1}$, а $\mathbb{U}^{+} \in \Omega_{C, r}^{+}-$оператор с предъядром $U$. Проверка того, что $\left(\mathbb{U}_{2}^{+} \mathbb{U}_{1}^{+}\right) f_{0}=\mathbb{U}^{+} f_{0}$, не представляет труда. Справедлива 
ЛЕмма 3.1. Класс $\Omega_{C, r}^{+}$образует коммутативную алгебру. Предбядром произведения $\mathbb{U}_{1}^{+} \mathbb{U}_{2}^{+}$служит свертка $U_{1} * U_{2}$ предбядер сомножителей:

$$
U(x)=\int_{0}^{x} U_{1}(x-t) d U_{2}(t) \quad n p u \quad U_{k}(0)=0, \quad k=1,2 .
$$

ЗАмечАниЕ. Если отказаться от требования $U(0)=0$, накладываемого на предъядра операторов из $\Omega_{C, r}^{+}$, то предъядром произведения операторов будет служить свертка, определяемая согласно правилу (2.9).

Рассмотрим теперь класс $\Omega_{C}^{-}$. При умножении операторов из этого класса удобно, как и в случае $\Omega_{C}^{-}$, нормировать предъядра согласно условию $U(0)=0$.

ЛЕмма 3.2. Класс операторов $\Omega_{C}^{-}$образует коммутативнуло алгебру. Предбядром $U$ произведения $\mathbb{U}^{-}=\mathbb{U}_{2}^{-} \mathbb{U}_{1}^{-}$, где $\mathbb{U}_{1,2}^{-} \in \Omega_{C}^{-}$, служит свертка (3.1) предбядер сомножителей.

ДокаЗАТЕЛЬСТво. Пусть $f \in E^{+}, f=f_{0}+f_{1}$, где $f_{0}(x) \equiv f(\infty), f_{1}(\infty)=0$. Обозначим $\varphi=\mathbb{U}_{1}^{-} f_{1}$ и $\psi=\mathbb{U}_{2}^{-} \varphi$. Имеем

$$
\varphi(y)=\int_{y}^{\infty} f_{1}(t) d U_{1}(t-y), \quad \psi(x)=\int_{x}^{\infty} \varphi(y) d U_{2}(y-x) .
$$

Применяя в выражении $\varphi$ интегрирование по частям, с учетом $U_{1}(0)=$ $f_{1}(\infty)=0$ получаем

$$
\varphi(y)=-\int_{y}^{\infty} U_{1}(t-y) d f_{1}(y) .
$$

Поэтому

$$
\psi(x)=-\int_{x}^{\infty}\left[\int_{y}^{\infty} U_{1}(t-y) d f_{1}(t)\right] d_{y} U_{2}(y-x) .
$$

Поменяв порядок интегрирования, получаем

$$
\psi(x)=-\int_{x}^{\infty} U(t-x) d f_{1}(t)=\int_{x}^{\infty} f_{1}(t) d U(t-x)
$$

где $U$ определяется согласно (3.1). Легко проверить, что $\left(\mathbb{U}_{2}^{-} \mathbb{U}_{1}^{-}\right) f_{0}=\mathbb{U}^{-} f_{0}$. Лемма доказана.

Примечательно, что правило композиции предъядер в $\Omega_{C}^{+}$и $\Omega_{C}^{-}$одинаково.

2. Нижнее уравнение восстановления. Рассмотрим уравнение вида (0.5) на произвольном промежутке $[0 ; r), r \leqslant+\infty: \varphi=g+\mathbb{U}^{+} \varphi$,

$$
\varphi(x)=g(x)-\int_{0}^{x} \varphi(t) d U(x-t), \quad U \in V_{C}[0 ; r] .
$$

Это уравнение назовем нижним уравнением восстановления (УВ). Пусть $\gamma_{r} \equiv \bigvee_{0}^{r} U \quad(<+\infty)$. Согласно $(2.1)$ имеем $\left\|\mathbb{U}^{+}\right\| \leqslant \gamma_{r}$. Если $\gamma_{r}<1$, то оператор $\mathbb{U}^{+}$сжимаюший в $E[0 ; r]$ и $(3.2)$ обладает единственным решением в $E[0 ; r]$ при 
$g \in E[0 ; r]$. Пусть теперь $r<+\infty$ и $\gamma_{r} \geqslant 1$. Путем одного элементарного преобразования этот случай можно свести к случаю сжимающего оператора. Для этого умножим обе части (3.2) на $e^{-p x}, p>0$; получаем

$$
\widetilde{\varphi}(x)=\tilde{g}(x)-\int_{0}^{x} \widetilde{\varphi}(t) e^{-p(x-t)} d U(x-t),
$$

где $\widetilde{\varphi}(x)=e^{-p x} \varphi(x), \tilde{g}(x)=e^{-p x} g(x)$.

Пусть $\mathbb{U}_{p}$ - оператор вида

$$
\psi(x) \equiv\left(\mathbb{U}_{p} \varphi\right)(x)=-\int_{0}^{x} \varphi(t) e^{-p(x-t)} d U(x-t) .
$$

Очевидно, что $\mathbb{U}_{p}$ ограничен в $E[0 ; r]$. Покажем, что $\left\|\mathbb{U}_{p}\right\|_{E[0 ; r]}<1$ при достаточно большом $p$. Обозначим

$$
\rho(x)=\bigvee_{0}^{x} U, \quad \rho \in \operatorname{Mon}^{C}[0 ; r] .
$$

Выберем число $\delta>0$ так, чтобы $\rho(\delta) \leqslant 1 / 4$. Далее выберем число $p>0$ так, чтобы $e^{-p \delta}<1 /\left(4 \gamma_{r}\right)$. Покажем, что тогда $\left\|\mathbb{U}_{p}\right\| \leqslant 1 / 2$. Мы ограничимся доказательством этой оценки в $C[0 ; r]$. Пусть $\varphi \in C[0 ; r],\|\varphi\|=M$, а $\psi=U_{p} \varphi$ определяется согласно (3.4). Из (3.4) имеем следуюшее: если $x \leqslant \delta$, то

$$
|\psi(x)| \leqslant \int_{0}^{x} M d \rho(x-t) \leqslant M \rho(\delta) \leqslant \frac{1}{4} M .
$$

Пусть теперь $x>\delta$. Тогда

$$
|\psi(x)| \leqslant M \int_{0}^{\delta} d \rho(t)+M \int_{\delta}^{x} e^{-p \delta} d \rho(t) \leqslant \frac{1}{2} M .
$$

Из полученных неравенств следует $\left\|\mathbb{U}_{p} \varphi\right\| \leqslant \frac{1}{2}\|\varphi\|$, поэтому $\left\|\mathbb{U}_{p}\right\|_{C[0 ; r]} \leqslant \frac{1}{2}$.

Уравнение (3.2) нами заменено уравнением (3.3) со сжимающим оператором $\mathbb{U}_{p} ;(3.3)$ обладает единственным решением $\widetilde{\varphi} \in E[0 ; r]$, поэтому $(3.2)$ имеет единственное решение $\varphi(x)=e^{p x} \widetilde{\varphi}(x) \in E[0 ; r]$.

В силу $\left\|\mathbb{U}_{p}\right\|<1$ последовательные приближения для (3.3) сходятся. Определим итерации для (3.3) согласно соотношениям

$$
\widetilde{\varphi}_{n+1}=\tilde{g}+\mathbb{U}_{p} \widetilde{\varphi}_{n}, \quad \widetilde{\varphi}_{0}=0, \quad n=0,1, \ldots
$$

Рассмотрим также итерации для (3.2)

$$
\varphi_{n+1}=g+\mathbb{U}^{+} \varphi_{n}, \quad \varphi_{0}=0, \quad n=0,1, \ldots
$$

Итерации $\varphi_{n}$ и $\widetilde{\varphi}_{n}$ связаны простым соотношением $\varphi_{n}(x)=e^{p x} \widetilde{\varphi}_{n}(x)$. Поэтому из сходимости $\left(\varphi_{n}\right)$ в $E[0 ; r], r<\infty$, следует сходимость $\left(\varphi_{n}\right)$ в этих пространствах.

Пусть теперь $U \in V_{C}^{\text {loc }}[0 ; \infty)$ и $g \in E^{\mathrm{loc}}[0 ; \infty)$. Тогда согласно доказанному вьше (3.2) обладает единственным решением $\varphi \in E[0 ; r]$ на произвольном конечном промежутке $[0 ; r]$. Очевидно, что при $r^{\prime}>r$ решение, построенное на $[0 ; r]$, является продолжением решения на $[0 ; r]$. Поэтому (3.2) обладает единственным решением в $E^{\text {loc }}[0 ; \infty)$, причем итерации $(3.6)$ сходятся к этому решению по топологии $E^{\mathrm{loc}}[0 ; \infty)$. Нами доказана 
Лемма 3.3. а) При $U \in V_{C}[0 ; r] u g \in E[0 ; r], r<+\infty$, УВ (3.2) обладает единственным решением $\varphi \in E[0 ; r]$, причем $\varphi$ явлется пределом в $E[0 ; r]$ итерачий (3.6).

б) При $\gamma=\bigvee_{0}^{\infty} U<1$ и $g \in E^{+}$УВ (3.2) обладает единственным решением $\varphi \in E^{+}$, являющ,имся пределом итераций (3.6) в $E^{+}$.

в) При $U \in V_{C}^{\mathrm{loc}}[0 ; \infty)$ u $g \in E^{\mathrm{loc}}[0 ; \infty)$ УВ (3.2) обладает единственнылм решением в $E^{\operatorname{loc}}[0 ; \infty]$, причем итерации (3.6) сходятся $\kappa$ этому решению по топологии пространства $E^{\mathrm{loc}}[0 ; \infty)$.

г) Ecли $U \in \operatorname{Mon}^{C}[0 ; r)$ u $g \geqslant 0$ или $g \in \operatorname{Mon}^{C}[0 ; r)$, mo $\varphi \geqslant 0$ или $\varphi \in$ $\operatorname{Mon}[0 ; r), r \leqslant+\infty$.

Утверждения леммы 3.3 нетрудно перефразировать в терминах обратимости оператора $I-\mathbb{U}^{+}$, где $I$ - единичньй оператор, и положительности обратного оператора $\left(I-\mathbb{U}^{+}\right)^{-1}$ по конусу неотрицательных или монотонных функций.

Рассмотрим вопрос о построении оператора $\left(I-\mathbb{U}^{+}\right)^{-1}$ в виде $I+\boldsymbol{\Gamma}_{+}$, где $\boldsymbol{\Gamma}_{+} \in$ $\Omega_{C, r}^{+}$. Предъядро Г искомого оператора $\Gamma_{+}$назовем резольвентны.м предбядром. Из равенства

$$
\left(I-\mathbb{U}^{+}\right)^{-1}=I+\Gamma_{+}
$$

следует, что Г определяется из УВ

$$
\Gamma(x)=U(x)-\int_{0}^{x} \Gamma(t) d U(x-t) .
$$

Легко убедиться, что разрешимость $(3.8)$ в $V_{C}^{+}$является необходимым и достаточным условием обратимости оператора $I-\mathbb{U}^{+}$в пространстве $E^{+}$. Что касается пространства $V_{C}^{\text {loc }}[0 ; \infty)$, то согласно лемме 3.3 уравнение $(3.8)$ всегда обладает решением в данном пространстве при $U \in V_{C}^{\mathrm{loc}}$.

Во всех случаях, предусмотренных леммой 3.3 , решение уравнения (3.2) задается формулой

$$
\varphi(x)=g(x)-\int_{0}^{x} g(t) d \Gamma(x-t) .
$$

3. УВ в консервативном случае. Под консервативным случаем (KC) уравнения (3.2) мы будем подразумевать выполнение условий

$$
U(0)=0, \quad U(+\infty)=1, \quad U(x) \uparrow \quad \text { по } \quad x .
$$

Эти условия согласованы с условием (0.8) и относятся к тому частному случаю, когда распределение случайной величины сосредоточено на положительной полуоси. Изучение УВ в консервативном случае представляет не только самостоятельный интерес, но и важно с точки зрения изучения однородного уравнения (0.7) в консервативном случае (0.8).

Для нас специальный интерес представляет изучение асимптотических и других свойств следующего уравнения в $\mathrm{KC}$ :

$$
S(x)=1-\int_{0}^{x} S(t) d U(x-t) .
$$

2 Серия математическая, №2 
В случае абсолютно непрерьвной функции $U: U^{\prime}(x)=W(x) \in L_{1}^{+}$, уравнение (3.11) обрашается в следуюшее уравнение, которое весьма хорошо изучено в связи с приложениями в теории вероятностей (теория восстановления), теории переноса излучения и др.:

$$
S(x)=1+\int_{0}^{x} W(x-t) S(t) d t .
$$

Классические методы изучения УВ (3.12) основаны на применении преобразования Лапласа и соответствуюших тауберовых теорем (см. [5]). В [10] использованы прямые методы изучения (3.12), допускающие применение к уравнению (3.11). Ниже мы исследуем уравнение (3.12), применяя при этом соответствующие модификации результатов и методов работы [10].

Используя формулу (3.9), получаем

$$
S(x)=1+\Gamma(x), \quad \gamma \leqslant 1
$$

т.е. мы фактически занимаемся изучением свойств резольвентного предъядра уравнения (3.2).

Покажем, что функция $S(x) \uparrow$ в $\mathrm{KC}$ не ограничена. Доказательство ведется от противного. Пусть $\lim _{x \rightarrow+\infty} S(x)=M<+\infty, \delta>0$ и числа $r_{1}, r_{2}>0$ удовлетворяют условиям

$$
U(x)>1-\delta \quad \text { при } \quad x>r_{1}, \quad S(x)>M-\delta \quad \text { при } \quad x>r_{2} .
$$

Из (3.11) при $x>r_{1}+r_{2}$ имеем

$$
S(x) \geqslant 1+\int_{0}^{r_{1}} S(x-t) d U(t) \geqslant 1+(M-\delta)(1-\delta),
$$

откуда $M \geqslant 1+(M-\delta)(1-\delta)$. В силу произвольности $\delta$ приходим к противоречию. Из неограниченности функции $S$ следует, что в КС оператор $I-\mathbb{U}^{+}$не обратим ни в одном из пространств $E^{+}$.

Одним из замечательных свойств решения $S$ уравнения (3.12) является следующее свойство полуаддитивности (см. [12], [10]):

$$
S(x+y) \leqslant S(x)+S(y), \quad x, y \geqslant 0 .
$$

Ниже нами будет показано, что аналогичным свойством обладает решение (3.11) в КС. Заменим в (3.11) $x$ на $x+y$, где $y$ - фиксированное число:

$$
S(x+y)=1+\int_{0}^{x} S(x+y-t) d U(t)+\int_{x}^{x+y} S(x+y-t) d U(t),
$$

откуда имеем

$$
\widetilde{S}(x) \leqslant 1+S(y) \int_{x}^{x+y} d U(t)+\int_{0}^{x} \widetilde{S}(x-t) d U(t),
$$

где $\widetilde{S}(x)=S(x+y)$. Нами использован тот факт, что если $t \in[x ; x+y]$, то $S(x+$ $y-t) \leqslant S(y)$. Пусть функция $\varphi$ удовлетворяет УВ

$$
\varphi(x)=S(y) \int_{x}^{x+y} d U(t)-\int_{0}^{x} \varphi(t) d U(x-t) .
$$

Используя итерации вида (3.6) для (3.16) с учетом $\gamma=1$, легко убедиться, что $\varphi(x) \leqslant S(y)$. Учитывая положительность оператора $\left(I-\mathbb{U}^{+}\right)^{-1}$, от интегрального неравенства (3.15) приходим к неравенству (3.14). 
4. Асимптотические свойства решения уравнения (3.11) в диссипативном и консервативном случаях. Ниже мы будем заниматься изучением асимптотики при $x \rightarrow+\infty$ решения $S$ УВ (3.11) как в КС (3.10), так и в диссипативном случае (ДС), когда

$$
U(0)=0, \quad U(+\infty)=\gamma<1, \quad U(x) \uparrow \quad \text { по } \quad x .
$$

Начнем с рассмотрения ДС. Тогда в силу $\gamma<1$ оператор $I-\mathbb{U}^{+}$обладает положительньм обратным в $C[0 ; \infty]$, причем $S$ - неотрицательная, непрерывная, неубывающая, ограниченная функция на $[0 ; \infty), \exists \lim _{x \rightarrow+\infty} S(x)=M$. Вычислим этот предел. Используя равенство (2.7), можно совершить предельньй переход в (3.11) при $x \rightarrow+\infty: M=1+\gamma M$, откуда

$$
\lim _{x \rightarrow+\infty} S(x)=(1-\gamma)^{-1}, \quad \gamma<1 .
$$

Перейдем к рассмотрению УВ (3.11) в КС. Покажем, что $\exists$ числа $a, b>0$ такие, что

$$
S(x) \leqslant a x+b .
$$

Так как $S$ является пределом итераций типа (3.6) для (3.11), то для получения оценки (3.19) достаточно подобрать числа $a$ и $b$ так, чтобы выполнялось неравенство

$$
1+\int_{0}^{x}(a t+b) d U(x-t) \leqslant a x+b
$$

С учетом $\gamma=1$ неравенство (3.20) сводится к неравенству

$$
(a x+b) \int_{x}^{\infty} d U(t)+a \int_{0}^{x} t d U(t) \geqslant 1 .
$$

Пусть число $r>0$ удовлетворяет условиям

$$
\alpha \equiv \int_{0}^{r} t d U(t)>0, \quad \beta \equiv \int_{r}^{\infty} d U(t)>0 .
$$

Пусть $a=\alpha^{-1}$ и $b=\beta^{-1}$. Тогда левая часть (3.21) будет $\geqslant 1:$ при $x \leqslant r$ за счет первого слагаемого, а при $x>r$ за счет второго слагаемого.

Как и в случае уравнений (3.12), для (3.11) можно получить более точную асимптотику функции $S$, используя первый момент $m_{1}$ меры $d U$. Обозначим

$$
m_{1}=\int_{0}^{\infty} t d U(t) \leqslant+\infty
$$

Рассмотрим сначала случай $m_{1}=+\infty$. Ясно, что тогда $\forall \varepsilon>0$ можно взять число $r$ настолько большим, чтобы $\alpha>\varepsilon^{-1}$. Поэтому в оценке (3.19) число $a=\alpha^{-1}$ может быть сколь угодно малым. Поэтому при $m_{1}=+\infty$ имеем

$$
S(x)=o(x), \quad x \rightarrow+\infty .
$$


Пусть теперь $m_{1}<+\infty$. Обозначим $q(x)=S(x)-x / m_{1}$. Функция $q$ удовлетворяет УВ

$$
q(x)=q_{0}(x)-\int_{0}^{x} q(t) d U(x-t)
$$

где $q_{0}(x)=\frac{1}{m_{1}} \int_{x}^{\infty}(t-x) d U(x)$. Имеем $0 \leqslant q_{0} \leqslant 1$ и $q_{0}(x) \rightarrow 0$ при $x \rightarrow+\infty$. Покажем, что $q(x)=o(S(x))$ при $x \rightarrow+\infty$. Используя формулу $(3.9)$, получаем

$$
q(x)=q_{0}(x)-\int_{0}^{x} q_{0}(t) d \Gamma(x-t) .
$$

Пусть $\varepsilon>0$ и $q_{0}(x)<\varepsilon$ при $x>r$. Полагая в $(3.23) x>r$, будем иметь

$$
q(x) \leqslant \varepsilon-\int_{0}^{r} d_{t} \Gamma(x-t)-\int_{r}^{x} \varepsilon d \Gamma(x-t) \leqslant \varepsilon S(x)+[\Gamma(x)-\Gamma(x-r)] .
$$

С учетом полуаддитивности (3.14) функции $S(x)=\Gamma(x)+1$ получаем

$$
q(x) \leqslant \varepsilon S(x)+\Gamma(r)+1 \quad \text { при } \quad x>r .
$$

В силу произвольности числа $\varepsilon>0$ получаем

$$
q(x)=o(S(x)), \quad S(x)-\frac{x}{m_{1}}=o(S(x)), \quad x \rightarrow+\infty
$$

отсюда при $m_{1}<+\infty$ следует асимптотика

$$
S(x)=\frac{x}{m_{1}}+o(x), \quad x \rightarrow+\infty
$$

Объединяя результаты предыдушего и настояшего пунктов по уравнению (3.11), мы приходим к следующей теореме.

ТЕОРемА 3.1. В КС (3.10) решение $S$ уравнения восстановления (3.11) обладает следующими свойствами:

а) $S(x) \geqslant 1$ - непрерывная, монотонно неограниченно возрастающая функиия на $[0 ; \infty)$; имеет место соотношение (3.13);

б) $S$ обладает свойством полуаддитивности (3.14);

в) nусть $m_{1} \equiv \int_{0}^{\infty} t d U(t) \leqslant+\infty$; nри $m_{1}<+\infty$ имеет место асимптоти$\kappa a$ (3.24), при $m_{1}=+\infty$ имеет место асимптотика (3.22);

г) в диссипативном случае (3.17) решение $S$ УВ (3.11) является положительной, непрерывной, неубывающей, ограниченной функиией на $[0 ; \infty)$, предел которой в $\infty$ определяется согласно (3.18); имеет место формула (3.13).

Заметим, что сформулированные в теореме 3.1 свойства решения УВ (3.11) отличаются от известных свойств решения УВ (3.12) (см. [5], [10]) только тем, что решение УВ (3.12) является абсолютно непрерывным, а решение УВ (3.11) является непрерывным и имеет сингулярную компоненту, если такую компоненту имеет функция $U$. 
5. Верхнее уравнение восстановления. Перейдем к изучению следующего уравнения:

$$
\left(I-\mathbb{U}^{-}\right) \varphi=g, \quad \mathbb{U}^{-} \in \Omega_{C}^{-}, \quad g \in E^{+},
$$

или, в раскрытом виде,

$$
\varphi(x)=g(x)+\int_{x}^{\infty} \varphi(t) d U(t-x), \quad U \in V_{C}^{+} .
$$

Уравнение (3.26) назовем верхним уравнением восстановления.

Обозначим $\gamma \equiv \bigvee_{0}^{\infty} U<+\infty$. При $\gamma<1$ оператор $\mathbb{U}^{-}$, согласно (2.12), сжимающий в $E^{+}$и $I-\mathbb{U}^{-}$обратим в $E^{+}$. Ищем $\left(I-\mathbb{U}^{-}\right)^{-1}$ в виде $I+\boldsymbol{\Gamma}_{-}$, где $\boldsymbol{\Gamma}_{-} \in \Omega_{C}^{-}$. Используя правило композиции предъядер в $\Omega_{C}^{-}$(см. лемму 3.2 ), для Г получаем уравнение

$$
\Gamma(x)=U(x)-\int_{0}^{x} \Gamma(t) d U(t-x) .
$$

Если (3.27) обладает решением $\Gamma \in V_{C}^{+}$, то с его помошью решение УВ (3.26) записывается в виде

$$
\varphi(x)=g(x)+\int_{x}^{\infty} g(t) d \Gamma(t-x) .
$$

Сходство между верхним и нижним уравнениями восстановления (3.28) и (3.2) проявляется в том, что их резольвентные предъядра удовлетворяют одному и тому же уравнению. Однако есть и принципиальная разница между указанными УВ: $(3.2)$ всегда однозначно разрешимо в $E^{\text {loc }}[0 ; \infty)$ при $U \in V_{C}^{\mathrm{loc}}[0 ; \infty)$ и $g \in E^{\text {loc }}[0 ; \infty)$, причем тогда справедлива формула (3.9), а вопрос разрешимости (3.28) и применимости формулы (3.28) нуждается в специальном исследовании. Приведем один интересный пример нетривиальной разрешимости однородного уравнения (3.26) (c $g=0)$. В консервативном случае (3.10) указанное уравнение обладает очевидным нетривиальным решением $\varphi(x)=$ const. Этот факт будет нами использован в $\S 6$ для решения однородного уравнения $(0.7)$ в КС.

Что касается формулы (3.28), то она не столь универсальна, как (3.9), тем не менее с ее помощью можно построить решение УВ (3.26) в ряде случаев, когда оператор $I-\mathbb{U}^{-}$необратим в пространствах $E^{+}$и $\Gamma \in V_{C}^{+}$. В случае абсолютно непрерывного предъядра ряд результатов в указанном направлении по консервативному уравнению (3.26) содержится в [10]. Эти результаты частично допускают распространение на случай $U \in V_{C}^{+}$. В настоящей работе этот вопрос мы рассматривать не будем.

\section{§4. Задача факторизации. Нелинейное уравнение факторизации}

1. Одно мультипликативное свойство класса $\Omega_{C}$. В $\S 3$ нами было отмечено, что классы операторов $\Omega_{A}$ и $\Omega_{C}$ не замкнуты относительно умножения. Класс $\Omega_{A}$ обладает следуюшим замечательным свойством: если $\mathbb{U}^{ \pm} \in \Omega_{A}^{ \pm}$, то $\mathbb{U}^{-} \mathbb{U}^{+} \in \Omega_{A}$. Этот факт впервые был явно сформулирован и использован в $[7]$ и стал основой метода нелинейных уравнений факторизации (НУФ) автора для интегральных операторов Винера-Хопфа (см. [7], [10], [13]). На этом свойстве 
интегральных операторов Винера-Хопфа, в неявной форме, основаны также ранее известные факторизационные методы решения интегральных уравнений Винера-Хопфа (ИУВХ). Ниже нами будет показано, что аналогичным свойством обладает класс $\Omega_{C}$. На его основании предлагается метод НУФ для уравнений (0.1) с непрерывным предъядром, содержашим сингулярную компоненту.

Прежде всего отметим, что если с точки зрения изучения и решения УВ (3.2) и (3.26) было удобно нормировать предъядра согласно условию $U(0)=0$, то в целях изучения факторизационной задачи мы в дальнейшем будем нормировать эти предъядра согласно условию $U(\infty)=0$. Нами будут использованы "новые" предъядра $W_{ \pm}$для операторов из $\Omega_{C}^{ \pm}$, связанные со старыми предъядрами соотношением $W(x)=U(\infty)-U(x)$. Тогда

$\mathbb{U}^{+} f(x)=\int_{0}^{x} f(t) d W_{+}(x-t), \quad \mathbb{U}^{-} f(x)=\int_{x}^{\infty} f(t) d W_{-}(t-x), \quad W_{ \pm}(\infty)=0$.

Пусть операторы $\mathbb{U}^{ \pm} \in \Omega_{C}^{ \pm}$записаны в терминах новых предъядер $W_{ \pm}$, a $f \in E^{+}$. Обозначим $\varphi=\mathbb{U}^{+} f, \psi=\mathbb{U}^{-} \varphi=H f$, где $\mathbb{H}=\mathbb{U}^{-} \mathbb{U}^{+}$. Имеем

$$
\varphi(y)=\int_{0}^{y} f(t) d W_{+}(y-t), \quad \psi(x)=-\int_{x}^{\infty} \varphi(y) d W_{-}(y-x)
$$

Производя в выражении для $\varphi$ интегрирование по частям, получаем

$$
\varphi(y)=f(0) W_{+}(y)-\int_{0}^{y} \widetilde{W}_{+}(y-t) d f(t), \quad \widetilde{W}_{+}(y)=W_{+}(y)-W_{+}(0) .
$$

Подставляя полученное выражение для $\varphi$ в выражение для $\psi$, будем иметь

$$
\begin{aligned}
\psi(x) & =-A-\int_{x}^{\infty} d W_{-}(y-x) \int_{0}^{y} \widetilde{W}_{+}(y-t) d f(t), \\
A & =f(0) \int_{x}^{\infty} \widetilde{W}_{+}(y) d W_{-}(y-x) .
\end{aligned}
$$

Изменяя порядок интегрирования, будем иметь

$$
\begin{aligned}
\psi(x) & =-A-\int_{0}^{\infty} d f(t)\left[\int_{\max (x, t)}^{\infty} \widetilde{W}_{+}(y-x) d W(y-t)\right] \\
& =-A-\int_{0}^{\infty} d f(t)\left[\int_{\max (t-x, 0)}^{\infty} \widetilde{W}_{+}(x-t+z) d W_{-}(z)\right] .
\end{aligned}
$$

Интегрирование по частям приводит к следующему выражению:

$$
\psi(x)=\mathbb{H} f(x)=\int_{0}^{\infty} f(t) d h(x-t), \quad h(x)=\int_{\max (-x, 0)}^{\infty} \widetilde{W}_{+}(x+z) d W_{-}(z) .
$$

Ниже мы разложим оператор $\mathbb{H}$ по подпространствам $\Omega_{C}^{ \pm}$, используя при этом новые предъядра для $\mathbb{H}^{ \pm}$. При $x \geqslant 0$ имеем

$$
h(x)=\int_{0}^{\infty} W_{+}(x+z) d W_{-}(z)+c .
$$


С учетом $h^{+}(\infty)=0$ получаем

$$
h^{+}(x)=\int_{0}^{\infty} W_{+}(x+z) d W_{-}(z) .
$$

При $x \leqslant 0$ имеем

$$
h(x)=\int_{-x}^{\infty} \widetilde{W}_{+}(x+z) d W_{-}(z)=\int_{0}^{\infty} \widetilde{W}_{+}(t) d W_{-}(t-x) .
$$

Интегрируя по частям, с учетом $\widetilde{W}_{+}(0)=W_{-}(\infty)=0$ получаем

$$
h(x)=\int_{0}^{\infty} W_{-}(t-x) d W_{+}(x),
$$

откуда следует

$$
h^{-}(x)=\int_{0}^{\infty} W_{-}(x+t) d W_{+}(t), \quad x \geqslant 0 .
$$

Нами доказана следующая основная лемма.

Лемма 4.1. Если $\mathbb{U}^{ \pm} \in \Omega_{C}^{ \pm}$, mо $\mathbb{H} \equiv \mathbb{U}^{-} \mathbb{U}^{+} \in \Omega_{C}$, причем $\mathbb{H}=\mathbb{H}^{+}+\mathbb{H}^{-}$,

$$
\begin{gathered}
\mathbb{H}^{+} f(x)=\int_{0}^{x} f(t) d h^{+}(x-t), \quad \mathbb{H}^{-} f(x)=-\int_{x}^{\infty} f(t) d h^{-}(t-x), \\
h^{ \pm}(x)=\int_{0}^{\infty} W_{ \pm}(x+t) d W_{\mp}(t), \quad x \geqslant 0, \quad W_{ \pm}(\infty)=0 .
\end{gathered}
$$

2. Задача факторизации. Центральным вопросом настоящей работы является следующая задача факторизации: для $\mathbb{T} \in \Omega_{C}$ найти $\mathbb{U}^{ \pm} \in \Omega_{C}^{ \pm}$такие, чтобы имело место разложение

$$
I-\mathbb{T}=\left(I-\mathbb{U}^{-}\right)\left(I-\mathbb{U}^{+}\right) .
$$

Факторизация (4.4) понимается как равенство операторов, действуюших в $E^{+}$. Равенство (4.4) эквивалентно равенству $\mathbb{U}^{+}+\mathbb{U}^{-}=\mathbb{T}+\mathbb{U}^{-} \mathbb{U}^{+}$. Согласно лемме 4.1 $\mathbb{H}=\mathbb{U}^{-} \mathbb{U}^{+} \in \Omega_{C}$ и данное равенство эквивалентно следуюшим двум равенствам: $\mathbb{U}^{ \pm}=\mathbb{T}_{ \pm}+\mathbb{H}^{ \pm}$, где $\mathbb{H}^{ \pm}$определены согласно лемме 4.1. Перейдя к равенству предъядер, с учетом (4.3) получаем

$$
\begin{aligned}
& W_{+}(x)=T_{+}(x)-\int_{0}^{\infty} W_{+}(x+t) d W_{-}(t), \\
& W_{-}(x)=T_{-}(x)-\int_{0}^{\infty} W_{-}(x+t) d W_{+}(t),
\end{aligned}
$$

где $T_{+}(x)=T(+\infty)-T(x), T_{-}(x)=T(-x), x \geqslant 0$.

Соотношения (4.5) мы назовем нелинейным уравнением факторизации (НУФ) для задачи (4.4).

Рассмотрим тот частньй случай, когда функция $T$ обладает свойством симметрии:

$$
T(x)+T(-x)=2 T(0) .
$$


Выполнение условия (4.6) назовем симметрическим случаем (СС). В случае, когда роль $T$ играет непрерывная фонкция распределения некоторой случайной величины, условие симметрии (4.6) означает, что эта случайная величина симметрично распределена относительно начала координат. При выполнении (4.6) имеем

$$
T_{+}(x)=T_{-}(x)=T_{0}(x)
$$

Очевидно, что если $W$ удовлетворяет уравнению

$$
W(x)=T_{0}(x)-\int_{0}^{\infty} W(x+t) d W(t), \quad W \in V_{C}^{+},
$$

то $W_{ \pm}=W$ удовлетворяет уравнению (4.5) при (4.6).

Нами доказана

ТЕоремА 4.1. а) Факторизащия (4.4) имеет место тогда и только тогда, когда “новие" предбядра $W_{ \pm}$операторов $\mathbb{W}^{ \pm}$удовлетворяют НУФ (4.5).

б) Если в симметрическом случае (4.6) функиия $W$ удовлетворяет уравнению (4.8), то имеет место факторизачия (4.4), причем тогда $W_{+}=$ $W_{-}=W$.

Уравнения (4.5) и (4.8) являются обобшением и новой формой записи НУФ интегральных операторов Винера-Хопфа (см. [7], [10]):

$$
V_{ \pm}(x)=K_{ \pm}(x)+\int_{0}^{\infty} V_{ \pm}(x+t) V_{\mp}(t) d t
$$

где $K_{ \pm}(x)=-T_{ \pm}^{\prime}(x)$ - заданные, а $V_{ \pm}-$искомые функции из $L_{1}^{+}$. Уравнения (4.9) сводятся к (4.4) путем введения новых искомых функций

$$
W_{ \pm}(x)=\int_{x}^{\infty} V_{ \pm}(t) d t
$$

Таким образом, НУФ (4.5) является обобшением (4.9), так как (4.5) охватьвает также случай предъядер из $\Omega_{C}$, не являющихся абсолютно непрерывными.

\section{§5. Основное решение НУФ}

1. Настоящий параграф посвящен доказательству существования одного специального решения НУФ (4.5) и его симметрического случая (4.8), а также изучению свойств этого решения при предположении, что предъядро $T$ удовлетворяет условиям

$$
T(-\infty)=0, \quad T(x) \uparrow \quad \text { по } \quad x, \quad T(+\infty)=\mu \leqslant 1 .
$$

Условия (5.1) охватывают консервативный случай $(\mathrm{KC}) \mu=1$ и диссипативный случай (ДС) $\mu<1$.

Рассмотрим следуюшие последовательные приближения для (4.5):

$$
W_{n+1}^{ \pm}(x)=T_{ \pm}(x)-\int_{0}^{\infty} W_{n}^{ \pm}(x+t) d W_{n}^{\mp}(t), \quad W_{0}^{ \pm}=0, \quad n=0,1, \ldots
$$


Выполнение условий (5.1) мы пока не будем предполагать. Из свойств операторов из $\Omega_{C}^{-}$(см. $\left.\S 2\right)$ следует, что итерации (5.2) определяют последовательности $W_{n}^{ \pm}$в $V_{C}^{+}$. При сходимости этих последовательностей в $V_{C}^{+}: W_{n}^{ \pm} \rightarrow W_{ \pm} \in V_{C}^{+}$, можно показать, что $\left(W_{+}, W_{-}\right)$является решением НУ $\Phi$ (4.5). Такое решение назовем основнылм решением (ОР) НУФ (4.5). Аналогичным образом определяется ОР для симметрического случая (4.8) НУФ как предел итераций, определяемых посредством

$$
W_{n+1}(x)=T_{0}(x)-\int_{0}^{\infty} W_{n}(x+t) d W_{n}(t), \quad W_{0}=0, \quad n=0,1, \ldots
$$

Легко проверить индукцией по $n$, что при вьполнении условия (4.7) имеют место равенства $W_{n}^{+}=W_{n}^{-}=W_{n}$. Поэтому в СС сушествование ОР уравнения (4.5) равносильно существованию ОР НУФ (4.8).

2. Существование ОР. Исследуем вопрос о существовании ОР уравнений (4.5) при выполнении условий (5.1). Заметим, что из монотонного возрастания функции $T$ следует убывание $T_{ \pm}$, причем $T_{ \pm}(x) \downarrow 0$ при $x \rightarrow+\infty$.

ЛЕмма 5.1. При выполнении условий (5.1) последовательности $W_{n}^{ \pm}\left(u W_{n}\right)$ обладают следующими свойствами:

a) $0 \leqslant W_{n}^{ \pm}(x)$ no $x, W_{n}^{ \pm} \in V_{C}^{+}$;

б) $W_{n}^{ \pm}(+\infty)=0$;

в) $W_{n}^{ \pm}$возрастают nо $n$;

г) функиии $\delta_{n}^{ \pm}(x)=W_{n+1}^{ \pm}(x)-W_{n}^{ \pm}(x)$ неотрицательны и убьввалт по $x$.

ДокАЗАТЕЛЬСТво. Утверждения а) и б) леммы проверяем индукцией по $n$, используя при этом лемму 2.3 о свойствах операторов из $\Omega_{C}^{-}$. Докажем утверждения в) и г) совместно индукцией по $n$. Имеем $\delta_{0}^{ \pm}(x)=T_{ \pm}(x)$; они неотрицательны и убывают по $x$. Пусть $0 \leqslant \delta_{n}^{ \pm}(x) \downarrow$ по $x, n \leqslant m$. Пусть $0 \leqslant x_{1}<x_{2}<+\infty$. Из (5.2) имеем

$$
\begin{aligned}
& W_{m+1}^{ \pm}\left(x_{2}\right)-W_{m+1}^{ \pm}\left(x_{1}\right)=T_{ \pm}\left(x_{2}\right)-T_{ \pm}\left(x_{1}\right) \\
& \quad-\int_{0}^{\infty}\left[W_{m}^{ \pm}\left(x_{2}+t\right)-W_{m}^{ \pm}\left(x_{1}+t\right)\right] d W_{m}^{\mp}(t), \\
& W_{m}^{ \pm}\left(x_{2}\right)-W_{m}^{ \pm}\left(x_{1}\right)=T_{ \pm}\left(x_{2}\right)-T_{ \pm}\left(x_{1}\right) \\
& \quad-\int_{0}^{\infty}\left[W_{m-1}^{ \pm}\left(x_{2}+t\right)-W_{m-1}^{ \pm}\left(x_{1}+t\right)\right] d W_{m-1}^{\mp}(t) .
\end{aligned}
$$

Согласно предположению индукции имеем

$$
0 \leqslant \delta_{m}^{ \pm}\left(x_{2}\right) \leqslant \delta_{m}^{ \pm}\left(x_{1}\right)
$$

что эквивалентно неравенству

$$
W_{m}^{ \pm}\left(x_{1}\right)-W_{m}^{ \pm}\left(x_{2}\right) \geqslant W_{m-1}^{ \pm}\left(x_{1}\right)-W_{m-1}^{ \pm}\left(x_{2}\right) .
$$

Отсюда следует, что меры $-d W_{m}^{ \pm}$больше или равны мерам $-d W_{m-1}^{ \pm}$. Из (5.5) с учетом сказанного следует соответствуюшее неравенство между левыми частями первого и второго из соотношений (5.4). Мы пришли к неравенству (5.5), в котором $m$ заменено на $m+1$. Лемма доказана. 
Обозначим: $\mu_{ \pm}=T_{ \pm}(0), \mu=\mu_{+}+\mu_{-}(\leqslant 1), \gamma_{n}^{ \pm}=W_{n}^{ \pm}(0)$. Числа $\mu_{ \pm}$ и $\gamma_{n}^{ \pm}$представляют собой нормы соответствуюших функций в пространстве $V_{C}^{+}$. Из утверждения а) леммы 5.1 следует, что $\gamma_{n}^{ \pm} \uparrow$ по $n$. Ниже будет установлена ограниченность этих последовательностей. Подставляя в (5.2) $x=0$, будем иметь

$$
\gamma_{n+1}^{+}=\mu_{+}-\int_{0}^{\infty} W_{n}^{+}(t) d W_{n}^{-}(t), \quad \gamma_{n+1}^{-}=\mu_{-}-\int_{0}^{\infty} W_{n}^{-}(t) d W_{n}^{+}(t) .
$$

Производя в последнем соотношении интегрирование по частям, с учетом $W_{n}^{ \pm}(\infty)=0$ получаем

$$
\gamma_{n+1}^{-}=\mu_{-}+\gamma_{n}^{-} \gamma_{n}^{+}+\int_{0}^{\infty} W_{n}^{+}(t) d W_{n}^{-}(t) .
$$

Складывая полученное равенство с (5.6), получаем

$$
\gamma_{n+1}^{+}+\gamma_{n+1}^{-}=\mu+\gamma_{n}^{-} \gamma_{n}^{+}
$$

Соотношение (5.7) идентично соотношениям, возникающим при построении основного канонического решения НУФ (4.9) для интегральных уравнений Винера-Хопфа (см. [7], [10]). Из (5.7) и монотонности $\gamma_{n}^{ \pm} \uparrow$ легко следуют оценки

$$
\gamma_{n}^{ \pm} \leqslant \mu \quad(\leqslant 1)
$$

Действительно, перепишем (5.7) в виде

$$
\gamma_{n+1}^{ \pm}=\mu-\left(\gamma_{n+1}^{\mp}-\gamma_{n}^{\mp}\right)-\gamma_{n}^{\mp}\left(1-\gamma_{n}^{ \pm}\right),
$$

откуда получаем (5.8) индукцией по $n$. Итак, последовательности $\gamma_{n}^{ \pm}$возрастают и ограничены, поэтому они сходятся: $\gamma_{n}^{ \pm} \rightarrow \gamma_{ \pm} \leqslant \mu$. Из (5.7) следует

$$
\left(1-\gamma_{-}\right)\left(1-\gamma_{+}\right)=1-\mu, \quad \gamma_{ \pm} \leqslant \mu .
$$

Нами показано, что $W_{n}^{ \pm}(x) \geqslant 0$ образуют возрастаюшие последовательности монотонно убываюших непрерывных функций, причем

$$
0=W_{n}^{ \pm}(\infty) \leqslant W_{n}^{ \pm}(x) \leqslant W_{n}^{ \pm}(0)=\gamma_{n}^{ \pm} \leqslant \gamma_{ \pm} \leqslant \mu .
$$

Полагая в неравенствах (5.5) $x_{1}=0, x_{2}=x$, получаем

$$
\delta_{n}^{ \pm}(x)=W_{n+1}^{ \pm}(x)-W_{n}^{ \pm}(x) \leqslant \gamma_{n+1}^{ \pm}-\gamma_{n}^{ \pm} .
$$

Поэтому $W_{n}^{ \pm}$монотонно и равномерно сходятся к монотонным функциям $W_{ \pm} \in$ $V_{C}^{+}, W_{ \pm}(x) \downarrow$ по $x$, причем $W_{ \pm}(\infty)=0$.

Докажем законность предельного перехода в (5.2). Пусть

$$
\sigma_{n}^{ \pm}(x)=\int_{0}^{\infty} W_{n}^{ \pm}(x+t) d W_{n}^{\mp}(t), \quad \sigma_{ \pm}(x)=\int_{0}^{\infty} W_{ \pm}(x+t) d W_{\mp}(t) .
$$

Имеем

$$
\begin{aligned}
\sigma_{ \pm}(x)-\sigma_{n}^{ \pm}(x)= & \int_{0}^{\infty}\left[W_{ \pm}(x+t)-W_{n}^{ \pm}(x+t)\right] d W_{\mp}(t) \\
& +\int_{0}^{\infty} W_{n}^{ \pm}(x+t) d\left[W_{\mp}(t)-W_{n}^{\mp}(t)\right],
\end{aligned}
$$

откуда нетрудно получить неравенство

$$
\left|\sigma_{ \pm}(x)-\sigma_{n}^{ \pm}(x)\right| \leqslant\left(\gamma_{ \pm}-\gamma_{n}^{ \pm}\right) \gamma_{\mp}+\gamma_{n}^{ \pm}\left(\gamma_{\mp}-\gamma_{n}^{\mp}\right) \rightarrow 0 .
$$

В симметрическом случае имеем $W_{n}^{ \pm}=W_{n}$,

$$
\gamma_{n}^{ \pm}=\gamma_{n} \uparrow \gamma=1-\sqrt{1-\mu} .
$$

Нами доказана 
ТЕОРема 5.1. При выполнении условий (5.1) НУФ (4.5) и (4.8) обладают основными решениями $\left(W_{+}, W_{-}\right)$и $W$ соответственно, причем:

а) $W_{ \pm} \in V_{C}^{+}, 0 \leqslant W_{ \pm}(x) \downarrow 0$ при $x \rightarrow+\infty$; имеют место равенство $u$ оценки (5.9);

б) в симметрическом случае (4.7) ОР НУФ (4.8) обладает дополнительныцм свойством (5.10);

в) существует факторизачия (4.4), где $\mathbb{U}^{ \pm} \in \Omega_{C}^{ \pm}$суть операторь вида (4.1), причем $\left(W_{+}, W_{-}\right)$является ОР НУФ (4.5) или НУФ (4.8) - в СС.

3. Дальнейшее изучение факторизации (4.4) с помощью моментов предъядра $T$ в КС. В настоящем и последующем пунктах будут сформулированы две леммы о свойствах факторизации (4.4) в КС. Эти леммы представляют собой обобщение леммы 4.6 и теоремы 4.1 работы [10], относящихся к факторизации интегральных операторов Винера-Хопфа. Доказательства нижеприводимых лемм 5.2 и 5.3 несущественно отличаются от доказательств соответствуюших результатов работы [10]. Поэтому мы ограничимся изложением доказательства только леммы 5.3, а лемму 5.2 приведем без доказательства.

Рассмотрим факторизацию (4.4) в КС. В силу $\mu=1$ согласно (5.9) имеем $\left(1-\gamma_{-}\right)\left(1-\gamma_{+}\right)=0$ (и $\left.\gamma_{ \pm} \leqslant 1\right)$. Поэтому в КС хотя бы одно из чисел $\gamma_{ \pm}$равно 1. Из результатов $\S 3$ известно, что равенство единице числа $\gamma_{+}$или $\gamma_{-}$означает необратимость соответственно операторов $I-\mathbb{U}^{+}$или $I-\mathbb{U}^{-}$. Первым выводом из этих фактов является тот, что в КС хотя бы один из факторов $I-\mathbb{U}^{ \pm}$, участвуюших в факторизации (4.4), необратим, стало быть, необратим и сам оператор $I-\mathbb{T}$. Вопрос о том, какое из чисел $\gamma_{ \pm}$равно 1 в $\mathrm{KC}$, представляет большой интерес в вопросах разрешимости однородного и неоднородного консервативных уравнений $(0.1)-$ см. $\S 6$. Ясно, что в СС (4.7) $\gamma_{+}=\gamma_{-}=1$, т.е. оба фактора в правой части (4.4) необратимы.

Предположим, что сходятся интегралы

$$
\nu_{ \pm}=-\int_{0}^{\infty} x d T_{ \pm}(x)<+\infty
$$

Обозначим

$$
\nu_{1}=\nu_{+}-\nu_{-}=\int_{-\infty}^{\infty} x d T(x) .
$$

Число $\nu_{1}$ представляет собой математическое ожидание случайной величины с функцией распределения $T$. Справедлива

ЛЕмма 5.2. а) Если $\nu_{1}>0$, mo $\gamma_{+}=1, \gamma_{-}<1$.

б) $E c л и \nu_{1}<0, m o \gamma_{+}<1, \gamma_{-}=1$.

в) $E c л и \nu_{1}=0, m o \gamma_{ \pm}=1$.

4. Вычисление первого момента функции $W$ в консервативном

CC. Пусть $\nu_{2} \leqslant+\infty$ - второй момент меры $d T$, а $m_{1}-$ первый момент меры $-d U$ :

$$
\nu_{2}=\int_{-\infty}^{\infty} x^{2} d T(x) \leqslant+\infty, \quad m_{1}=-\int_{0}^{\infty} x d U(x) \leqslant+\infty
$$


ЛЕмма 5.3. а) Если в симметрическом $K C \nu_{2}<+\infty$, то

$$
m_{1}=\sqrt{\nu_{2} / 2} .
$$

б) Если же $\nu_{2}=+\infty$, mo $m_{1}=+\infty$.

ДоказАтельство. Пусть $\nu_{2}<+\infty$. Покажем сначала, что $m_{1} \leqslant \sqrt{\nu_{2} / 2}$. Рассмотрим итерации (5.3). Покажем индукцией по $n$, что все функции $W_{n}$ обладают конечными моментами второго порядка $m_{2}^{(n)}$. Действительно, пусть $m_{2}^{(n)}<+\infty$. Применяя к (5.3) формулу (2.13) при $h(x)=x^{2}$ и $r=+\infty$, получаем

$$
m_{2}^{(n+1)}=\frac{1}{2} \nu_{2}+\int_{0}^{\infty}\left[\int_{0}^{\infty} x^{2} d W_{n}(x+t)\right] d W_{n}(t)
$$

или

$$
m_{2}^{(n+1)}=\frac{1}{2} \nu_{2}+\int_{0}^{\infty}\left[\int_{t}^{\infty}(x-t)^{2} d W_{n}(x)\right] d W_{n}(t)
$$

откуда имеем

$$
m_{2}^{(n+1)} \leqslant \frac{1}{2} \nu_{2}+m_{2}^{(n)} \gamma_{n}<+\infty
$$

Изменяя в (5.12) порядок интегрирования и меняя местами $x$ и $t$, получаем

$$
m_{2}^{(n+1)}=\frac{1}{2} \nu_{2}+\int_{0}^{\infty}\left[\int_{0}^{x}(x-t)^{2} d W_{n}(x)\right] d W_{n}(t) .
$$

Складывая (5.12) и (5.13) и деля пополам, получаем

$$
m_{2}^{(n+1)}=\frac{1}{2} \nu_{2}+\frac{1}{2} \int_{0}^{\infty}\left[\int_{0}^{\infty}(x-t)^{2} d W_{n}(x)\right] d W_{n}(t)
$$

откуда имеем

$$
m_{2}^{(n+1)}=\frac{1}{2} \nu_{2}+m_{2}^{(n)} \gamma_{n}-\left[m_{1}^{(n)}\right]^{2}, \quad m_{1}^{(n)}=-\int_{0}^{\infty} x d W_{n}(x)<+\infty,
$$

или

$$
\left[m_{1}^{(n)}\right]^{2}=\frac{1}{2} \nu_{2}-\left[m_{2}^{(n+1)}-m_{2}^{(n)} \gamma_{n}\right] \leqslant \frac{1}{2} \nu_{2},
$$

что влечет за собой неравенство $m_{1} \leqslant \sqrt{\nu_{2} / 2}$.

Докажем теперь обратное неравенство $m_{1} \geqslant \sqrt{\nu_{2} / 2}$. Применяя к равенству (4.8) формулу (2.13) с $h(x)=x^{2}$ и $r<+\infty$, получаем

$$
\int_{0}^{r} x^{2} d W(x)=\int_{0}^{r} x^{2} d T_{0}(x)-A,
$$

где

$$
A=\int_{0}^{\infty}\left[\int_{0}^{r} x^{2} d W(x+t)\right] d W(t)=\int_{0}^{\infty}\left[\int_{t}^{r+t}(x-t)^{2} d W(x)\right] d W(t)
$$


Имеем

$$
A \geqslant \int_{0}^{r}\left[\int_{t}^{r}(x-t)^{2} d W(x)\right] d W(t)=\int_{0}^{r}\left[\int_{r}^{x}(x-t)^{2} d W(t)\right] d W(x),
$$

поэтому

$$
A \geqslant \frac{1}{2} \int_{0}^{r}\left[\int_{0}^{r}(x-t)^{2} d W(x)\right] d W(t)=[1-W(r)] \int_{0}^{r} x^{2} d W(x)-\left[\int_{0}^{r} x d W(x)\right]^{2} .
$$

Используя полученное неравенство, из (5.14) будем иметь

$$
\int_{0}^{r} x^{2} d W(x) \geqslant \frac{1}{2} \int_{-r}^{r} x^{2} d T(x)+[1-W(r)] \int_{0}^{r} x^{2} d W(x)-\left[\int_{0}^{r} x d W(x)\right]^{2}
$$

или

$$
\left[-\int_{0}^{r} x d W(x)\right]^{2} \geqslant \frac{1}{2} \int_{-r}^{r} x^{2} d T(x) .
$$

Из полученного неравенства непосредственно следует как неравенство $m_{1} \geqslant$ $\sqrt{\nu_{2} / 2}$ при $\nu_{2}<+\infty$, так и утверждение б) леммы. Лемма доказана.

\section{§6. Уравнение $(0.1)$ в консервативном случае}

1. Настояший параграф посвящен применению факторизации (4.4) к решению уравнения (0.1) и соответствующего однородного уравнения $(0.7)$ в КС $(0.8)$.

Факторизация (4.4) сводит уравнение (0.1) к последовательному решению следуюших двух: верхнего и нижнего уравнений восстановления

$$
\left(I-\mathbb{U}^{-}\right) f=g, \quad\left(I-\mathbb{U}^{+}\right) \varphi=f,
$$

или, в раскрытом виде,

$$
\begin{aligned}
& f(x)=g(x)+\int_{x}^{\infty} f(t) d U_{-}(t-x), \\
& \varphi(x)=f(x)-\int_{0}^{x} \varphi(t) d U_{+}(x-t),
\end{aligned}
$$

где $U_{ \pm}(x)=W_{ \pm}(0)-W_{ \pm}(x)$.

В п. $3 \S 5$ было показано, что в $\mathrm{KC} \mathrm{оператор} I-\mathbb{T}$ необратим в пространствах $E^{+}$, т.е. случай (0.8) является особым случаем для уравнения (0.1). В качестве одного из наиболее интересных приложений факторизации (4.4) в особом $\mathrm{KC}$ мы ниже будем исследовать вопрос о сушествовании положительного решения однородного уравнения (0.7) в КС. Эта задача возникает в теории вероятностей, в теории очередей и др. как задача о нахождении максимума последовательных частичных сумм для одинаково распределенных независимых случайных величин (см. [2], [4]). В случае, когда $T$ - абсолютно непрерывная функция, т.е. распределение $T$ обладает плотностью $T^{\prime}(x)=K(x)$, задача $(0.7),(0.8)$ принимает вид

$$
\begin{gathered}
S(x)=\int_{0}^{\infty} K(x-t) S(t) d t \\
0 \leqslant K(x) \in L_{1}(\mathbb{R}), \quad \int_{-\infty}^{\infty} K(x) d x=1 .
\end{gathered}
$$


Различным частным случаям задачи (6.3), (6.4) посвящено огромное число исследований в связи с известной проблемой Милна в теории переноса излучения, с приложениями в кинетической теории газов, в теории вероятностей и др. (см. [2], $[4],[7],[10],[14])$. Что касается задачи $(0.7),(0.8)$ в случае $T_{S} \neq 0$, то результаты по ней в литературе нами не были обнаружены.

В работе [4] использовано понятие $\mathbb{P}$-решения и $\mathbb{P}^{*}$-решения задачи $(6.3),(6.4)$. Введем аналогичные понятия для задачи $(0.7),(0.8)$. Под $\mathbb{P}$-решением этой задачи будем понимать неотрицательную, неубывающую, непрерывную слева функцию на $[0 ; \infty)$, для которой $\lim _{x \rightarrow+\infty} S(x)=1$. Под $\mathbb{P}^{*}$-решением будем понимать неотрищательную, неубывающую, непрерьвную слева функцию с $\lim _{x \rightarrow+\infty} S(x)=$ $+\infty$.

Классические результаты по существованию $\mathbb{P}$-решения или $\mathbb{P}^{*}$-решения задачи $(6.3),(6.4)$ получены в работах [2], [4]. Наиболее общие результаты в указанном направлении получены в [10] на основании метода НУФ. Отметим, что задача о построении $\mathbb{P}^{*}$-решения является намного сложнее задачи о построении $\mathbb{P}$-решения (см. [4]).

Рассмотрим уравнения (6.1), (6.2), соответствующие однородному уравнению (0.8) в $\mathrm{KC}$,

$$
\begin{aligned}
& f(x)=\int_{x}^{\infty} f(t) d U_{-}(t-x) \\
& S(x)=f(x)-\int_{0}^{x} S(t) d U_{+}(x-t),
\end{aligned}
$$

где $U_{ \pm}(x)=W_{ \pm}(0)-W_{ \pm}(x)=\gamma_{ \pm}-W_{ \pm}(x)$.

Предположим, что выполняется некоторое условие, обеспечивающее выполнение равенства $\gamma_{-}=1$. Так обстоит дело в СС (4.7), когда $\gamma_{ \pm}=\gamma=1$ или при $\nu \leqslant 0$ (см. лемму 5.2). Из равенства $\gamma_{-}=1$ следует, что однородное уравнение (6.5) обладает нетривиальным решением $f(x) \equiv 1$. Поэтому уравнение (6.6) принимает вид (3.11). Согласно результатам $\S 3$ это уравнение обладает решением как при $\gamma_{+}<1$, так и при $\gamma_{+}=1$, причем имеет место формула (3.13). Свойства решения $S$ сфформулированы в теореме 3.1 .

Строго говоря, априори мы не можем гарантировать, что построенное нами решение $S$, полученное из уравнений $(6.5),(6.6)$, удовлетворяет исходному уравнению, поскольку при $\gamma_{+}=1$ построенная функция $S$ неограничена, а факторизация (4.4) имеет место как равенство операторов, действующих в классах ограниченных функций $E^{+}$. Аналогичная ситуация возникла в работе [10] в связи с решением уравнения (6.3), (6.4). Как и в работе [10], можно доказать, что решение факторизованного уравнения $(6.5),(6.6)$ удовлетворяет исходному уравнению (0.7), (0.8). Справедливы следуюшие теоремы.

ТЕОРема 6.1. Пусть предбядро однородного уравнения (0.7) обладает свойством симметрии (4.7). Тогда задача (0.7), (0.8) обладает $\mathbb{P}^{*}$-решением $S$, причем:

а) $S(x) \geqslant 1$ - непрерывная, неограниченно монотонно возрастающая функиия на $[0 ; \infty)$, удовлетворяющая условию полуаддитивности (3.4);

б) если $\nu_{2} \equiv \int_{-\infty}^{\infty} x^{2} d T(x)<+\infty$, то имеет место асимптотика

$$
S(x)=\frac{1}{\sqrt{\nu_{2} / 2}} x+o(x), \quad x \rightarrow+\infty
$$


если жсе $\nu_{2}=+\infty$, mo

$$
S(x)=o(x), \quad x \rightarrow+\infty
$$

Для полного доказательства теоремы нам остается заметить, что асимптотики (6.7) и (6.8) следуют из (3.22) и (3.24) с учетом леммы 5.3. Теорема доказана.

ТЕОРемА 6.2. Пусть предбядро консервативного однородного уравнения (0.7), (0.8) обладает конечным моментом первого порядка $\nu_{1}=$ $\int_{-\infty}^{\infty} x d T(x)$. Тогда:

а) в случае $\nu=0$ задача обладает $\mathbb{P}^{*}$-решением $S$; это решение обладает свойствами утверждения а) теоремь 6.1 и асимптотикой

$$
S(x)=O(x), \quad x \rightarrow+\infty
$$

б) если $\nu<0$, то задача обладает $\mathbb{P}$-решением $S_{1} \in \operatorname{Mon}^{C}[0 ; \infty], S_{1}(x)=$ $\left(1-\gamma_{+}\right) S(x)$, где $S$ определяется согласно (3.13); функиия $\Gamma=\Gamma_{+}$является резольвентным предбядром оператора $\mathbb{U}^{+}$, а число $\gamma_{+}<1$ - нормой оператора $\mathbb{U}^{+}$, әде $\mathbb{U}^{ \pm}$суть операторы, участвующие в факторизачии (4.4).

2. В настоящем пункте мы вкратце остановимся на рассмотрении неоднородного консервативного уравнения $(0.1)$, (0.8). Нами уже было отмечено, что факторизация (4.4) позволяет свести это уравнение к решению уравнений восстановления (6.1), (6.2). Мы ограничимся рассмотрением случая $\gamma_{-}<1, \gamma_{+}=1$. Тогда уравнение (6.1) обладает единственным решением в $E^{+}$при $g \in E^{+}$. Уравнение же (6.2) будет обладать единственным решением $S \in E^{\mathrm{loc}}[0 ; \infty)$, имеющим вид

$$
S(x)=f(x)-\int_{0}^{x} f(t) d \Gamma_{+}(x-t) .
$$

Из ограниченности функции $f \in E^{+}$и из результатов пп. $3,4 \S 3$ следует, что

$$
S(x)=O(x), \quad x \rightarrow+\infty
$$

\section{Нами доказана}

ТЕОрема 6.3. Если $\exists \nu_{1}=\int_{-\infty}^{\infty} x d T(x)$ и $\nu_{1}>0$, то уравнение $(0.1),(0.8)$ обладает решением из $E^{\mathrm{loc}}[0 ; \infty)$ с асимптотикой (6.11).

В заключение автор выражает глубокую благодарность академику В. С. Владимирову и участникам руководимого им семинара по математической физике МИРАН за ценное обсуждение результатов настоящей работы. 


\section{Список литературы}

1. Колмогоров A.H., Фомин C. В. Элементы теории функций и функционального анализа. М.: Наука, 1981.

2. Lindlay D. V. The theory of queue with a single server // Proc. Cambridge Phil. Soc. 1952. № 48. P. 277-289.

3. Крейн М. Г. Интегральные уравнения на полупрямой с ядрами, зависящими от разности аргументов // УМН. 1958. Т. 13. № 5. С. 3-120.

4. Spitzer F. The Wiener-Hopf Equation, whose kernel is a probability density // Duke Math. J. 1957. V. 24. № 3. P. 323-343.

5. Беллман P., Кук K. Дифференциально-разностные уравнения. М.: Мир, 1967.

6. Гохберг И. Ц., Фельдман И. А. Уравнения в свертках и проекционные методы их решения. М.: Наука, 1971.

7. Енгибарян Н. Б., Арутюнян А. А. Интегральные уравнения на полупрямой с разностными ядрами и нелинейные функциональные уравнения // Матем. сб. 1975. Т. 97. № 1. C. $35-58$.

8. Гахов Ф. Д., Черский Ю. И. Уравнения типа свертки. М.: Наука, 1978.

9. Владимиров В. С. Уравнение Винера-Хопфа в алгебрах Неванлинны и Смирнова // Изв. АН СССР. Сер. матем. 1987. Т. 51. № 4. С. 767-784.

10. Енгибарян Н. Б., Арабаджяя Л. Г. Уравнения в свертках и нелинейные функциональные уравнения // Итоги науки и техники. Мат. анализ. Т. 22. М.: ВИНИТИ АН СССР, 1984. C. $175-240$.

11. Спитцер $\Phi$. Принципы случайного блуждания. М.: Мир, 1969.

12. Феллер В. Введение в теорию вероятностей и ее приложения. Т. 2. М.: Мир, 1967.

13. Енгибарян H. Б. О нелинейных уравнениях факторизации операторов. Применение методов теории функций и функц. анализа к задачам мат. физики. Ереван: Изд-во АН АрмССР, 1982. С. 123-128.

14. Соболев В. В. Курс теоретической астрофизики. М.: Наука, 1967. 